SHORT REPORT

\title{
Frontal white matter anisotropy and symptom severity of late-life depression: a magnetic resonance diffusion tensor imaging study
}

\author{
K Nobuhara, G Okugawa, T Sugimoto, T Minami, C Tamagaki, K Takase, Y Saito, S Sawada, \\ T Kinoshita
}

\begin{abstract}
Objectives: To investigate the disruption of neural circuits in the frontal lobes and limbic structures in late-life depressed patients compared with healthy controls, and to examine the correlation between the degree of microstructural abnormalities of white matter and clinical symptom severity in latelife depression.

Methods: Thirteen patients with late-life depression and matched control subjects underwent diffusion tensor imaging. Fractional anisotropy (FA), an index of the integrity of white matter tracts, was determined in the white matter of frontal, temporal, and occipital brain regions and the corpus callosum.

Results: A significant reduction was found in white matter FA values of widespread regions of the frontal and temporal lobes of depressed patients. Also, there was some evidence suggesting that white matter FA values of the inferior frontal brain region are inversely related to severity of depression. Conclusions: These results suggest the possible loss of integrity within frontal and temporal white matter fibre tracts and implicate the orbitofrontal circuit in symptom severity in late-life depression.
\end{abstract}

$\mathrm{E}$ xecutive dysfunction, a disturbance associated with compromised integrity of frontal structures and their subcortical connections, has been reported in late-life depression. ${ }^{1}$ Disorders of the basal ganglia and their prefrontal projections are often complicated by depression and result in executive dysfunction. ${ }^{2}$ Magnetic resonance imaging (MRI) studies have found reduced volumes of the prefrontal lobes, hippocampus, and head of the caudate nucleus in late-life major depression. ${ }^{3}{ }^{4}$ Furthermore, postmortem studies have shown marked increases of deep white matter hyperintensities due to cerebral ischaemia, and a reduction in the packing density and number of glial cells in the dorsolateral prefrontal cortex of late-life depressed subjects. ${ }^{5}{ }^{6}$ Studies using diverse methods have also demonstrated abnormal brain structure and function in the frontal lobes and limbic structures of the patients with late-life depression. ${ }^{7}$

Magnetic resonance diffusion tensor imaging (DTI) of the brain reveals the orientation of white matter tracts in vivo and yields an index of microstructural integrity through quantification of the magnitude and directionality of restricted tissue water mobility (diffusion anisotropy) in three dimensions. ${ }^{8}$ Fractional anisotropy (FA) is a measure of the directionality of diffusion anisotropy. ${ }^{8}$ DTI has been used to discern the directionality of white matter tracts in the normal human brain ${ }^{9}$ and to evaluate the integrity of white matter fibres in psychiatric diseases such as schizophrenia ${ }^{10-12}$ and depression..$^{13} 14$

In the present study, we used DTI to examine disruption of the structural integrity of the white matter in patients with late-life depression compared with healthy controls. We also examined the relation between FA values of the frontal white matter regions and clinical symptoms in late-life depression.

\section{METHODS}

A total of 13 patients (four men, nine women; mean (SD) age 62.8 (6.6) years) who met DSM-IV criteria for unipolar major depression were enrolled in this study. We excluded patients with other axis I disorders including dementia, severe or acute medical illnesses (brain tumor, cardiac/hepatic/renal failure, and cardiac infarction within the last three months preceding the study), and neurological disorders (delirium, Parkinson's disease, and multiple sclerosis). We assessed cognitive function with the Mini-Mental State Examination (MMSE), and patients with MMSE scores lower than 25 were excluded to ensure that people with dementia were not included in the study. T2-weighted MRI of all patients did not show any white matter changes such as infarction or other vascular lesions. The patients had an average of one prior episode of depression with mean age of illness onset at 52.9 (7.3) years. The age of onset of nine subjects was 60 years or younger, and four patients developed depression after 60 years of age. The mean duration of illness was 4.0 (2.6) years. All patients were taking antidepressant medication: tricyclic antidepressants, or selective serotonin reuptake inhibitors, or serotonin noradrenaline reuptake inhibitors, or combination of these. We also recruited 13 age matched control subjects (four men, nine women; 61.5 (4.8) years). Depressive symptoms were assessed using the 17-item Hamilton Depression Rating Scale (HDRS). The mean HDRS score was $33.4(9.8)$ at the time of the study.

The protocol was approved by the Ethics Committee of Kansai Medical University Hospital, Osaka, Japan. All subjects gave informed consent before participating the study.

All subjects underwent DTI scanning, which can be implemented on conventional MR scanners. MR scans were taken with a $1.5 \mathrm{~T}$ General Electric Signa Horizon LX (GE Medical Systems, Milwaukee, WI). Head motion was minimised with restraining pads. Sagittal Tl-weighted images were acquired first, with a slice clearly displaying both the anterior commissure (AC) and the posterior commissure (PC). Then, a series of axial diffusion-weighted images with a diffusion sensitising gradient $\left(b\right.$ value $=1000 \mathrm{~s} / \mathrm{mm}^{2}$ ) were

Abbreviations: $\mathrm{AC} / \mathrm{PC}$, anterior commissure/posterior commissure; DTI, diffusion tensor imaging; FA, fractional anisotropy; HDRS, Hamilton Depression Rating Scale; ROI, region of interest 
obtained. Diffusion was measured along six non-colinear directions. We used single shot spin-echo echo-planar sequences for our diffusion tensor analysis. All of the acquisitions paralleled the AC-PC line using the following parameters: $\quad \mathrm{TR}=4000 \mathrm{msec}, \quad \mathrm{TE}=103 \mathrm{msec}, \quad 128 \times 128$ matrix, $\mathrm{FOV}=24 \times 24 \mathrm{~cm}, \mathrm{NEX}=4,6.0 \mathrm{~mm}$ slice thickness, $2.0 \mathrm{~mm}$ gap. The DTI acquisition time was less than five minutes.

The diffusion-weighted images were transferred to a dedicated workstation (Advantage Workstation; GE Medical Systems, Milwaukee, WI) where the DTI data were postprocessed using Functool 2.2.49 (GE Medical Systems, Milwaukee, WI). Echo-planar imaging (EPI) distortion was corrected automatically. The diffusion eigenvectors and eigenvalues $\left(\lambda_{1}, \lambda_{2}, \lambda_{3}\right)$, which correspond to the main direction of diffusion and associated diffusivity, were calculated from the diffusion tensor. FA, which reveals information about the degree of diffusion anisotropy in white matter, was then calculated as follows ${ }^{8}$ :

$$
F A=\sqrt{\frac{3}{2}} \frac{\sqrt{\left[\lambda_{1}-(\lambda)\right]^{2}+\left[\lambda_{2}-(\lambda)\right]^{2}+\left[\lambda_{3}-(\lambda)\right]^{2}}}{\sqrt{\lambda_{1}^{2}+\lambda_{2}^{2}+\lambda_{3}^{2}}}
$$

where

$$
(\lambda)=\frac{\lambda_{1}+\lambda_{2}+\lambda_{2}}{3}
$$

Circular regions of interest (ROI) were positioned bilaterally at $8 \mathrm{~mm}$ above the AC-PC plane, at the AC-PC plane, and at $8 \mathrm{~mm}$ below the AC-PC plane in the frontal white matter (fig 1). In addition, elliptical ROI were placed in the genu and splenium of the corpus callosum, as well as bilaterally in temporal, parietal, and occipital white matter. The ROI, except for the genu and splenium, were standardised at $88 \mathrm{~mm}^{2}$ to minimise partial volume effects from grey matter. The ROI for the genu and splenium of the corpus callosum adjacent to the lateral ventricles were of a different shape and smaller in size $\left(63 \mathrm{~mm}^{2}\right)$ to avoid effects from the cerebrospinal fluid. The diffusion tensor was computed for each voxel, and the eigenvalues and eigenvectors were determined. The FA was computed from the diffusion tensor.

The unit of DTI analysis was regional white matter FA. Intraindividual hemispheric differences and intergroup (patients $v$ control) differences in FA were examined with paired $t$ tests and independent $t$ tests, respectively. Spearman's rank correlation coefficient $\left(r_{\mathrm{s}}\right)$ was used to test the correlation between the DTI regional measures and HDRS scores.

\section{RESULTS}

There were no significant intraindividual hemispheric differences in white matter FA. Therefore, FA was averaged across hemispheres for each white matter region. Table 1 shows the result of comparison with normal controls. Significant reductions in white matter FA values were found in widespread regions of the frontal and temporal lobes of the patients with depression compared with those of the controls. White matter FA values of the parietal and occipital brain regions as well as the corpus callosum in the depressed patients did not significantly differ from the normal controls.

Higher frontal white matter FA values at $8 \mathrm{~mm}$ below the AC-PC line correlated significantly with lower HDRS scores $\left(r_{\mathrm{s}}=-0.58, \mathrm{p}=0.04\right)$. HDRS scores did not correlate

Table 1 Mean (SD) of fractional anisotropy values of the selected brain regions for patients with late-life depression and control subjects

\begin{tabular}{lccc}
\hline & $\begin{array}{c}\text { Patients } \\
(\mathbf{n}=13)\end{array}$ & $\begin{array}{c}\text { Controls } \\
(\mathbf{n}=13)\end{array}$ & p value* \\
\hline Frontal white matter & & & \\
$8 \mathrm{~mm}$ above AC-PC & $0.274(0.023)$ & $0.316(0.021)$ & $<0.01$ \\
$0 \mathrm{~mm}$ below AC-PC & $0.286(0.032)$ & $0.335(0.029)$ & $<0.01$ \\
$8 \mathrm{~mm}$ below AC-PC & $0.279(0.023)$ & $0.324(0.030)$ & $<0.01$ \\
Temporal white matter & $0.301(0.029)$ & $0.343(0.025)$ & 0.01 \\
Parietal white matter & $0.321(0.052)$ & $0.330(0.031)$ & 0.58 \\
Occipital white matter & $0.330(0.048)$ & $0.322(0.038)$ & 0.64 \\
Genu & $0.619(0.074)$ & $0.656(0.056)$ & 0.17 \\
Splenium & $0.718(0.082)$ & $0.748(0.041)$ & 0.25 \\
\hline & *Independent $t$ tests for non-paired data. & \\
AC/PC, anterior commissure/posterior commissure. & \\
\hline
\end{tabular}

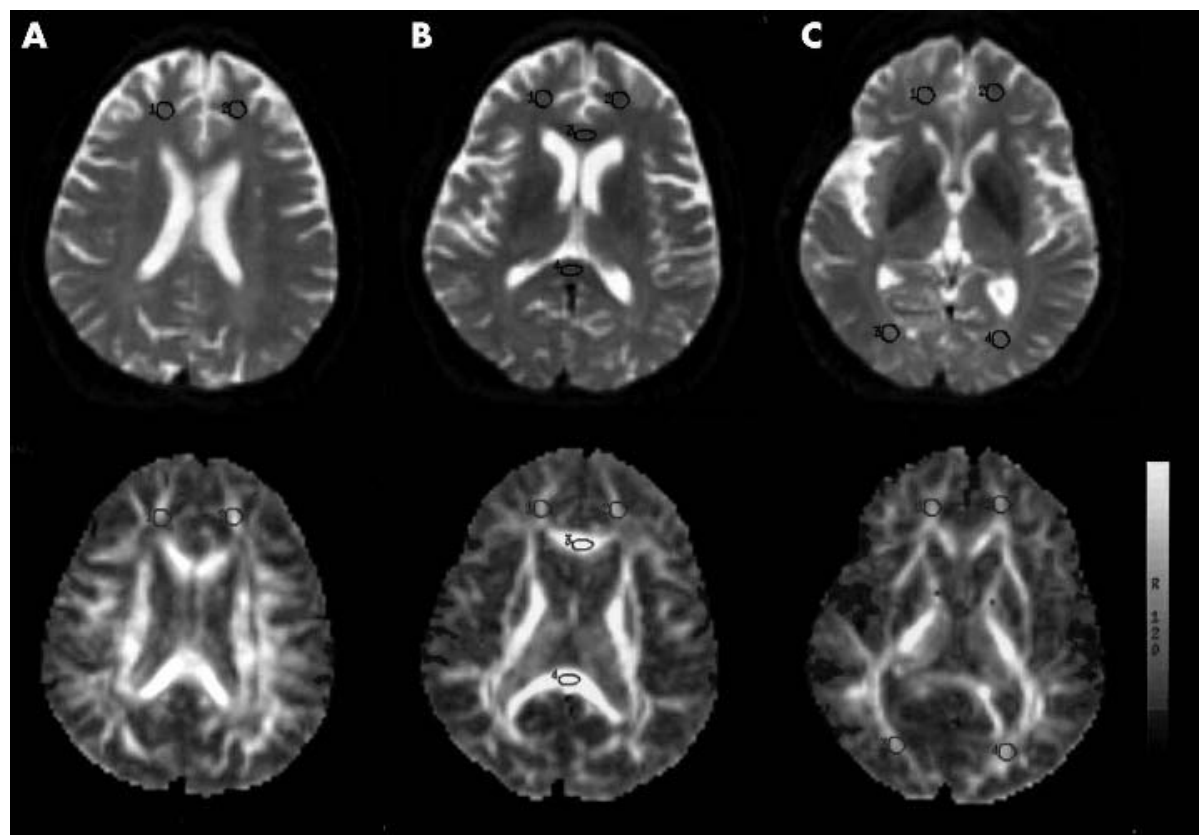

Figure 1 Horizontal T2-weighted images (top) and corresponding FA images (bottom) from a patient with late-life depression. Regions of interest (ROI) were drawn in the frontal white matter ( $\mathrm{ROI} 1$ and 2 ) at $8 \mathrm{~mm}$ above the anterior commissure (AC)-posterior commissure (PC) plane (A), frontal white matter (ROI 1 and 2), genu (ROI 3 ) and splenium (ROI 4) of the corpus callosum at the AC-PC plane (B), frontal white matter (ROI 1 and 2) and occipital white matter (ROI 3 and 4 ) at $8 \mathrm{~mm}$ below the AC-PC plane (C). 
significantly with either white matter $\mathrm{FA}$ values at $8 \mathrm{~mm}$ above the AC-PC line $\left(r_{\mathrm{s}}=-0.22, \mathrm{p}=0.48\right)$ or white matter FA values at the AC-PC line $\left(r_{\mathrm{s}}=0.12, \mathrm{p}=0.70\right)$. We did not correct these results for multiple tests because we were concerned about the risk of type II errors.

\section{DISCUSSION}

The primary result of our study is abnormal white matter anisotropy in widespread regions of the frontal and temporal lobes in patients with late-life depression. There was some evidence to suggest an inverse relation between white matter FA values of the inferior frontal brain region and the severity of depression.

Previous studies of DTI alterations in late-life depression have focused on the white matter of the frontal brain regions. ${ }^{13}{ }^{14}$ Taylor et $a l^{14}$ reported that microstructural changes in the white matter of the right superior frontal gyrus were associated with late-life depression. Alexopoulos et $a l^{13}$ showed that microstructural white matter abnormalities lateral to the anterior cingulate may be associated with a low rate of remission. The different parts of the frontal lobes have afferent and efferent connections with other neocortical, limbic, and subcortical regions and participate in the limbic-cortico-striatal-pallidal-thalamic circuits. ${ }^{7}$ These neuroanatomical circuits play an important role in the regulation and modulation of affect and emotion, and contribute to the pathogenesis of late-life depression.

The reduction of white matter anisotropy observed in our DTI study is suggestive of possible loss of integrity within frontal and temporal white matter fibre tracts, and supports the hypothesis that neuroanatomical circuit abnormalities are a key factor in the functional anatomy of late-life depression. Although white matter anisotropy could be influenced by many factors such as the dense packing of axons, relative membrane permeability to water, internal axonal structure, tissue water content, or degree of myelination, the pathophysiology underlying reduced white matter FA values in widespread regions of the frontal and temporal lobes in patients with late-life depression has not been confirmed. More than one process may be responsible for FA reduction.

The inferior frontal brain regions $(8 \mathrm{~mm}$ below the AC-PC plane) include the medial orbital prefrontal region and the neural pathways to caudate and other limbic regions. ${ }^{15}$ Smaller orbital frontal cortex volumes are associated with functional disability in late-life depression. ${ }^{16}$ Statistical parametric mapping analysis has shown increased lesion density in medial orbital prefrontal white matter and a correlation between severity of depression and lesions in the medial orbital region. ${ }^{17}$ Damage to the orbitofrontal circuit may lead to disinhibition, irritability, and diminished sensitivity to social cues. ${ }^{18}$ Our observations in the present study implicate the orbitofrontal circuit in the symptom severity in late-life depression.

The principal limitation of our study is the combination of a small sample size and multiple tests for correlations between the degree of macrostructural abnormalities of white matter and clinical symptom severity. In order not to reduce the power of the study further, we allowed the type II error rate to increase. Our patient population was relatively young for late-life depression. Also, we were unable to examine potential effects of antidepressants on DTI measures. Future studies will attempt to confirm the theories of neural circuit involvement in late-life depression and the contribution of microstructural changes in neuroanatomical circuits to the pathophysiology and outcome in late-life depression in larger numbers of patients.

\section{ACKNOWLEDGEMENTS}

We are grateful to the members of the MRI group at Kansai Medical University for valuable assistance. We also thank Akemi Saito and Gregory H Smith for their editorial assistance.

\section{Authors' affiliations}

K Nobuhara, G Okugawa, T Sugimoto, T Minami, C Tamagaki,

K Takase, Y Saito, T Kinoshita, Department of Neuropsychiatry, Kansai

Medical University, Moriguchi, Japan

S Sawada, Department of Radiology, Kansai Medical University, Moriguchi, Japan

This work was supported by a grant from the Ministry of Education, Science, and Culture of Japan (16591173).

Competing interests: none declared

Correspondence to: Dr Kenii Nobuhara, 10-15 Fumizono-cho Moriguchi City, 570-8506, Japan; nobuhara@takii.kmu.ac.jp

Received 29 September 2004

Revised version received 7 April 2005

Accepted 8 April 2005

\section{REFERENCES}

1 Lesser IM, Boone KB, Mehringer CM, et al. Cognition and white matter hyperintensities in older depressed patients. Am J Psychiatry 1996;153:1280-7.

2 Massman PJ, Delis DC, Butters N, et al. The subcortical dysfunction hypothesis of memory deficits in depression: neuropsychological validation in a subgroup of patients. J Clin Exp Neuropsychol 1992;14:687-706.

3 Kumar A, Bilker W, Jin Z, et al. Atrophy and high intensity lesions: complementary neurobiological mechanisms in late-life major depression. Neuropsychopharmacology 2000;22:264-74

4 Lloyd AJ, Ferrier IN, Barber R, et al. Hippocampal volume change in depression: late- and early-onset illness compared. Br J Psychiatry 2004; 184:488-95.

5 Thomas AJ, O'Brien JT, Davis S, et al. Ischemic basis for deep white matter hyperintensities in major depression: a neuropathological study. Arch Gen Psychiatry 2002;59:785-92.

6 Miguel-Hidalgo JJ, Baucom C, Dilley G, et al. Glial fibrillary acidic protein immunoreactivity in the prefrontal cortex distinguishes younger from older adults in major depressive disorder. Biol Psychiatry 2000;48:861-73.

7 Drevets WC. Geriatric depression: brain imaging correlates and pharmacologic considerations. J Clin Psychiatry 1994;55(Suppl A):71-81.

8 Basser PJ, Pierpaoli C. Microstructural and physiological features of tissues elucidated by quantitative-diffusion-tensor MRI. J Magn Reson B 1996; 111:209-19.

9 Abe O, Aoki S, Hayashi N, et al. Normal aging in the central nervous system: quantitative MR diffusion-tensor analysis. Neurobiol Aging 2002;23:433-41.

10 Lim KO, Hedehus M, Moseley M, et al. Compromised white matter tract integrity in schizophrenia inferred from diffusion tensor imaging. Arch Gen Psychiatry 1999;56:367-74.

11 Minami T, Nobuhara K, Okugawa G, et al. Diffusion tensor magnetic resonance imaging of disruption of regional white matter in schizophrenia. Neuropsychobiology 2003;47:141-5.

12 Okugawa G, Nobuhara K, Minami T, et al. Subtle disruption of the middle cerebellar peduncles in patients with schizophrenia. Neuropsychobiology 2004;50:119-23.

13 Alexopoulos GS, Kiosses DN, Choi SJ, et al. Frontal white matter microstructure and treatment response of late-life depression: a preliminary study. Am J Psychiatry 2002;159:1929-32.

14 Taylor WD, MacFall JR, Payne ME, et al. Late-life depression and microstructural abnormalities in dorsolateral prefrontal cortex white matter. Am J Psychiatry 2004;161:1293-6.

15 Carmichael ST, Price JL. Architectonic subdivision of the orbital and medial prefrontal cortex in the macaque monkey. J Comp Neurol 1994;346:366-402.

16 Taylor WD, Steffens DC, McQuoid DR, et al. Smaller orbital frontal cortex volumes associated with functional disability in depressed elders. Biol Psychiatry 2003;53:144-9.

17 MacFall JR, Payne ME, Provenzale JE, et al. Medial orbital frontal lesions in late-onset depression. Biol Psychiatry 2001;49:803-6.

18 George MS, Ketter TA, Post RM. Prefrontal cortex dysfunction in clinical depression. Depression 1994;2:59-72. 